\title{
Managing temporal uncertainty in multi-mode Z-number fuzzy graph structures
}

\author{
Margarita Knyazeva ${ }^{a}$ and Alexander Bozhenyuk ${ }^{b}$ and Uzay Kaymak ${ }^{c}$ \\ ${ }^{a}$ Southern Federal University, Taganrog, Russian Federation, mknyazeva@sfedu.ru \\ ${ }^{b}$ Southern Federal University, Taganrog, Russian Federation, avb002@yandex.ru \\ ${ }^{c}$ School of Industrial Engineering, Eindhoven University of Technology, The Netherlands, u.kaymak@ieee.org
}

\begin{abstract}
In this paper we introduce an NP-hard optimization problem and examine preferences of decision-maker towards imprecise alternatives (modes) in a fuzzy temporal graph structure model. Fuzzy Z-number preference matrix is introduced and types of generalized precedence relations in fuzzy multimode resource-constrained project scheduling problem (F-MRCPSP) based on expert estimation are discussed. Implementation of Z-numbers allows handling uncertain data and modelling preferences of expert towards uncertain variables. Multi-mode way for activity performance allows considering temporal uncertainty, expert estimations, flexibility in switching from mode to mode and resource levelling profile problem. Geometrical interpretation for fuzzy multi-mode problem ( $F$ $M R C P S P$ ) based on box packing is given.
\end{abstract}

Keywords: Fuzzy graph, Temporal uncertainty, Decision-making, Z-numbers, Scheduling, Geometrical interpretation, Topology, Modes, Preference matrix.

\section{Introduction}

The idea of implementing fuzzy graph theory models along with its extensions has been extensively studied due to its wide practical application in computer networks, chemistry, social network analysis, distributed systems, operations research and scheduling techniques. First Kaufmann [9], then Rosenfeld [16] gave the definitions of fuzzy graph and introduced the foundations in which the concept of fuzzy set is applied in graph structures with membership functions on nodes and arcs. Yeh and Bang [20] introduced a version of fuzzy graph to be applied in clustering analysis. Since then a number of authors studied different types of fuzziness possible in directed graphs [11], leading to a taxonomy of fuzzy graphs and fuzzy relations between elements. A practical application is reported in human trafficking [14].

Pairwise relations between elements in a graph can come along with some additional information, such as temporal constraints [3], topological ordering, weights or labels such as vertex coloring [2]. Temporal graphs, dynamic or time-varying graphs can be formally introduced as a special case of labeled graphs, where labels capture a measure of time or dynamic topology. For example in social networks, the topology can represent entities and relations between them. In transportation networks routes, units moving over and destination points can be modelled with the help of temporal relations and dynamic ordering of events. Finally, time-respecting paths or temporal paths whose edges use strictly increasing times can be considered and handled as a class of optimization scheduling problem. Temporal generalization of some elements in graphs structure affects complexity by introducing time-dependent variables. There are great variety of investigations concerning connectivity in static and dynamic graph structures. Classical Menger's theorem is one of the most basic theorems that represents the equivalence of max-flow min-cut theorem for undirected graphs. Its extension to the temporal case and single-labeled temporal graphs was investigated in [12].

The other interesting problem concerning temporal graphs and distributed dynamic network model was first introduced by Kuhn et al. [10] and after that presented by Dutta et al. [7] as the centralized algorithm for the problem of $k$-token dissemination in dynamic networks, where $k$-pieces of information in a distributed process should be disseminated to the all processes or participants of the system. The main idea of such algorithms, no matter centralized or distributed, is to consider the interaction between graph nodes in dynamic topology so that each node terminates and outputs all $k$-tokens. Another example of temporal 
extensions is classical travelling salesman problem in dynamic weighted graph, in which the nodes should be explored as soon as possible, the cost of an edge may vary in time and the goal is to find a minimum cost for temporal travelling salesman problem tour [13].

Temporal graphs, dynamic networks, recurrent or periodic graphs are obviously of particular interest due to their practical application in transportation systems, the underlying routing, allocating and scheduling problems.

In this paper we investigate complex interaction between elements in labelled temporal graph, modedependent states of a graph, topology in graph structure and suggest to consider uncertainty of expertestimated variables as a Z-fuzzy number preference matrix model. Additionally we introduce strongly NPhard fuzzy multi-mode resource-constrained project scheduling problem with generalized precedence relations $(F-M R C P S P)$ and its geometrical box packing interpretation as an extension of temporal graph problem.

\section{Modelling temporal intervals: problem formulation and complexity}

Let's consider a temporal fuzzy graph formulation and its extension to multi-mode resource-constrained project scheduling problem (F-MRCPSP). Multimode scheduling problem with generalized precedence activity-on-node (A-o-N) graph representation [15] can be defined as a number of activities (operations) that are to be scheduled according to their execution mode: time-dependent precedence relations, duration of activity, resources that are available and an objective function to optimize. Allocation of resources with respect to sequencing activities, constraints and objective function in diverse search space represents decision making process in scheduling problems.

Literature overview shows that there are different formulations of scheduling problems, each of them is usually based on 0-1 programming models, Boolean decision variables and satisfiability (SAT) problem solver [6]. In the case of general crisp temporal constraints the idea that some subsets of activities (nodes) with no precedence relations can be scheduled in parallel (feasible subsets) meeting the resource constraints, the problem formulation requires the definition of $O\left(2^{n} T\right)$ binary decision variables and $O\left(n^{2}, n T\right)$ restrictions. An extension of the problem to multi-mode formulation where each activity can be executed under different duration and corresponding resource usage with temporal generalized precedence relations causes the combination of subsets to be increased exponentially.
Our work is based on multi-mode resource-constrained project scheduling and fuzzy z-number duration estimation for activities that is made by expert. Mode assignment step is solved by constructing decision and preference matrices and it returns a current feasible mode assignment list that is used on the next scheduling step. We also consider flexibility that provides competitive value and ability to deal with uncertainty in its various manifestations in decision-making process. For example, temporal flexibility in the fuzzy starting times and durations of activities allows to take into account possible maximal and minimal overlaps between them; flexibility in the activity execution mode or multi-mode activity performance allows to consider trade-off between time and resource usage, costs, parallel activity execution and changes in product performance as well as other degree of freedom available during operational step of planning. Knapsack or rectangle packing interpretation of combinatorial F-MRCPSP of finding optimal graph-based schedule which minimizes the total project makespan, respects the precedence constraints and resource capacities for a given mode ${ }^{1}$ is given.

Definition 1. A scheduling project $\mathbf{P}$ is composed of four sets $P=\left\{\tilde{V}_{t m}, R, \operatorname{Pr}, M_{i}\right\}$, where $\tilde{V}_{t m}$ is a set of activities executed in one mode $m$ at $t$-moment, $R$ is a set of renewable resources available at every moment $t$ of a project performance horizon, set $\mathrm{Pr}$ denotes precedence constraints in set $\tilde{V}_{t m}$ and $M_{i}$ is a set of alternative execution modes for each activity $i \in \tilde{V}_{t m}$.

Definition 2. A directed fuzzy temporal graph is a quadruple $\tilde{G}=\left(\tilde{V}_{t m}, E, s, f\right)$, where $\tilde{V}_{t m}$ is a set of vertices/activities performed in mode $m$ at $t$-moment, $\tilde{V}_{t m}=\left\{\left(v_{1 t}, \mu\left(v_{1 t m}\right)\right),\left(v_{2 t}, \mu\left(v_{2 t m}\right)\right), \cdots,\left(v_{i t}, \mu\left(v_{i t m}\right)\right)\right\}$, and the set of edges $E=\left\{e_{1 m}, e_{2 m}, \cdots, e_{j m}\right\}$ reflects topological ordering as well as precedence relations between vertices; $s$ and $f: E \rightarrow V$ are two functions, $s$ being called the source function (start) and $f$ is the target function (endpoint) for every directed edge.

An example of $m$-mode activity-on-node graph representation is shown on Figure 1. Each activity on node $\tilde{V}_{t m}$ can be processed in different $m$-modes, where $m \in M_{i}$, and each activity has its fuzzy mode-dependent duration $\tilde{d}_{i m}$. Each fuzzy activity duration captures temporal intervals that can be proceeded one after another to get chain or so-called project makespan. Figure 1 illustrates three modes $M_{1}=\left\{m_{1}, m_{2}, m_{3}\right\}$ for activity 1 and the corresponding parallel mode-edges, i.e. the ways the activity can be executed. Switching from mode to mode provides flexibility during operational step of planning. In case

\footnotetext{
${ }^{1}$ Mode assignment step can be performed recursively in case no current feasible schedule can be found.
} 


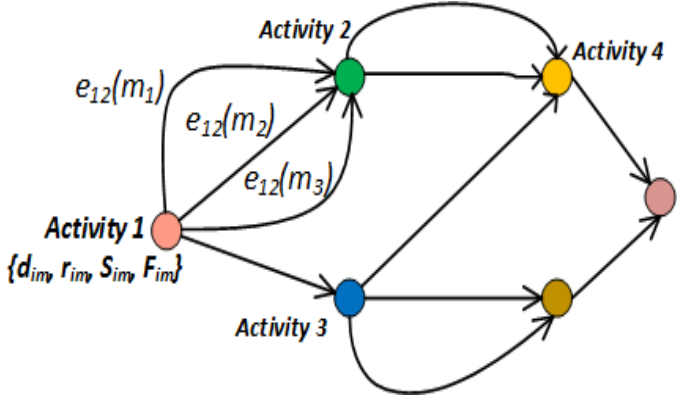

Figure 1: Activity-on-Node presentation of temporal multi-mode graph

there is uncertainty about temporal interval relations, each possible mode of activity execution is entered as an extra edge of a graph.

Definition 3. Given a directed fuzzy temporal graph $\tilde{G}=\left(\tilde{V}_{t m}, E, s, f\right)$ and a number of $m$-modes, two edges $e, e^{\prime} \in E$ are called parallel mode-edges iff $s(e)=s\left(e^{\prime}\right)$ and $t(e)=t\left(e^{\prime}\right)$ with $e$ being executed with mode $m$, and $e$ ' being executed in mode $m$ '.

Definition 4. Let $\mathrm{X}$ and $\mathrm{Y}$ be two fuzzy intervals in RCPSP. Then the followingrelations between temporal intervals can be formulated: before, after, meets, overlaps, during, starts, finishes (See Table 1). Relations between intervals are maintained in a fuzzy scheduling graph where vertices represent individual activities with certain fuzzy durations, start and finish times. Allen in his interval algebra [1] introduced seven types of relations between crisp temporal intervals and they can be used as spatial configuration in fuzzy scheduling graph structure to show topological finish-start precedence relations between vertices and sequencing order of activity execution.

\begin{tabular}{|c|c|c|}
\hline RELATION & SYMBOL & EXAMPLE \\
\hline X before Y & $\mathrm{i}$ & XXX YYY \\
X equal Y & $=$ & XXX \\
& $=$ & YYY \\
X meets Y & $\mathrm{m}$ & XXXYYY \\
X overlaps Y & $\mathrm{o}$ & XXX \\
& $\mathrm{o}$ & YYY \\
X during Y & $\mathrm{d}$ & XXX \\
& $\mathrm{d}$ & YYYYY \\
X starts Y & $\mathrm{s}$ & XXX \\
& $\mathrm{s}$ & YYYYYY \\
X finishes Y & $\mathrm{f}$ & XXX \\
& $\mathrm{f}$ & YYYYYY \\
\hline
\end{tabular}

Table 1: Types of relations on temporal intervals.

Let $i$ and $j$ be two activities, and $\tilde{S}_{i} ; \tilde{F}_{i}$ be a fuzzy starting and finishing times of activity $i, \tilde{d}_{i m}$ be a fuzzy duration of activity $i$ executed in mode $m ; t$ - discrete time unit. Based on start-finish relations on temporal intervals, eight different minimal and maximal temporal intervals for mode-dependent activities duration can be defined

$S S_{i j}^{m i n}$ and $S S_{i j}^{\max }$ minimal and maximal time intervals between start to start times of activity $i$ and activity $j$;

$S F_{i j}^{\min }$ and $S F_{i j}^{\max }$ minimal and maximal time intervals between start to finish times of activity $i$ and activity $j$;

$F S_{i j}^{\min }$ and $F S_{i j}^{\max }$ minimal and maximal time intervals between finish to start times of activity $i$ and activity $j$;

$F F_{i j}^{\min }$ and $F F_{i j}^{\max }$ minimal and maximal time intervals between finish to finish times of activity $i$ and activity $j$;

An example of start to start geometrical interpretation of $m$-mode activity temporal start to start intervals and precedence relations is presented on Figure 2.

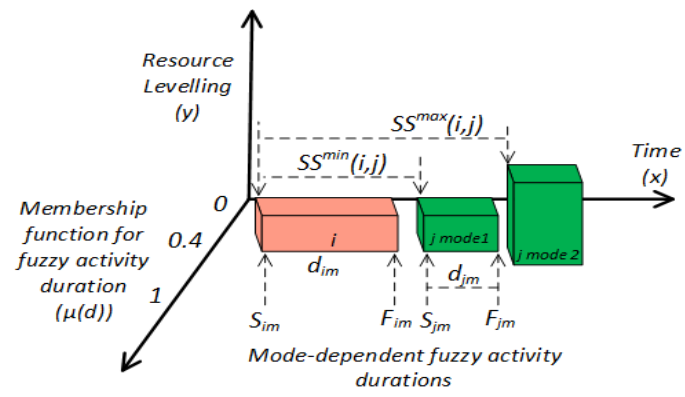

Figure 2: Example of minimal and maximal time intervals between start to start times of activities $i$ and $j$

Let's also consider some notions for the classical math programming problem formulation:

$\tilde{d}_{i}$ is a fuzzy estimated duration of activity $i$;

$\tilde{S}_{i}$ is a fuzzy start time of activity $i$;

$\tilde{e s} s_{i}$ and $\tilde{l s}_{i}$ are earliest and latest fuzzy starting times of activity $i$;

$R_{k}$ is a maximum number of resource type- $k$ available per period;

$r_{i m k}$ is a resource requirement of $k$-type for activity $i$ executed in mode $m$;

$K$ is the number of resource types required for activity execution;

$M_{i}$ is the set of $m$-modes available for $i$-activity execution;

$\tilde{T}$ is a shortest fuzzy project makespan, while $\bar{T}$ is an upper bound on the project duration;

$\tilde{E}_{S S}, \tilde{E}_{S F}, \tilde{E}_{F S}$ and $\tilde{E}_{F F}$ are defined as the resulting sets of fuzzy temporal intervals: set of early fuzzy start to start times, start to finish, finish to start and finish to finish intervals of activities execution correspond- 
ingly. Fuzzy finish time of activity execution can be calculated using the following equation:

$$
\tilde{F}_{i}=\tilde{S}_{i}+\tilde{d}_{i}
$$

Depending on the mode, intervals of fuzzy temporal variables may vary from minimal to maximal within a certain expert estimation membership level. In order to meet general precedence relations in fuzzy graph and to define feasible set of vertices to be scheduled linear programming problem formulation approaches have been developed by different authors [15]. Equation (1) refers to binary decision variable $x_{i m t}$ and in case of $m$-mode activity execution performance it can check if an activity $i$ is performed in mode $m$ and started in time $t$ :

$$
\begin{gathered}
x_{i m t}= \begin{cases}1, & \text { if } i \text { is performed in } m \text { at } t \\
0, & \text { otherwise. }\end{cases} \\
\sum_{t=\tilde{e_{s}}}^{\tilde{l s}_{n}} t x_{n t} \rightarrow \text { Min }
\end{gathered}
$$

subject to

$$
\begin{aligned}
& \sum_{m_{i}=1}^{M_{i}} \sum_{t=\tilde{e s} s_{i}}^{\tilde{l s_{i}}} x_{i m t}=1, i=1,2, \ldots, n \\
& \sum_{m_{i}=1}^{M_{i}} \sum_{t=\tilde{e s} s_{i}}^{\tilde{l s_{i}}}\left(t+S S_{i j}^{\text {min }}\right) x_{i m t} \leq \sum_{m_{j}=1}^{M_{j}} \sum_{t=\tilde{e s} s_{j}}^{\tilde{l s_{j}}} t x_{j m t} \\
& \langle i, j\rangle \in E_{S S} \\
& \sum_{m_{i}=1}^{M_{i}} \sum_{t=\tilde{e} s_{i}}^{\tilde{l s}_{i}}\left(t+S F_{i j}^{m i n}\right) x_{i m t} \leq \\
& \sum_{m_{j}=1}^{M_{j}} \sum_{t=\tilde{e}_{j}}^{\tilde{l s}_{j}}\left(t+\tilde{d}_{j m}\right) x_{j m t} \\
& \langle i, j\rangle \in E_{S F} \\
& \sum_{m_{i}=1}^{M_{i}} \sum_{t=\tilde{e} s_{i}}^{\tilde{l s_{i}}}\left(t+\tilde{d}_{i m}+F S_{i j}^{m i n}\right) x_{i m t} \leq \\
& \sum_{m_{j}=1}^{M_{j}} \sum_{t=\tilde{e s}_{j}}^{\tilde{l s}_{j}} t x_{j m t} \\
& \langle i, j\rangle \in E_{F S} \\
& \sum_{m_{i}=1}^{M_{i}} \sum_{t=\tilde{e s} s_{i}}^{\tilde{l s_{i}}}\left(t+\tilde{d}_{i m}+F F_{i j}^{m i n}\right) x_{i m t} \leq \\
& \sum_{m_{j}=1}^{M_{j}} \sum_{t=\tilde{e s}_{j}}^{\tilde{l s}_{j}}\left(t+\tilde{d}_{j m}\right) x_{j m t} \\
& \langle i, j\rangle \in E_{F F}
\end{aligned}
$$

$$
\begin{array}{r}
\sum_{i=1}^{n} \sum_{m_{i}=1}^{M_{i}} r_{i m k} \sum_{s=\max \left(t-\tilde{d}_{i m}, \tilde{e s}_{i}\right)}^{\min \left(t-1, \tilde{s_{i}}\right)} x_{i m s} \leq R_{k}, \\
k=1,2, \ldots, K ; t=1,2, \ldots, T ; x_{i m t} \in\{0,1\} \\
i=1,2, \ldots, n ; t=\tilde{e s} s_{i}, \ldots, \tilde{l s} s_{i} .
\end{array}
$$

In the following zero-one mathematical formulation we transform maximal and minimal time-lags between temporal intervals for activity performance into fuzzy interpretation. In this case maximal time lags and overlaps can be transformed into equivalent minimal time lags with a negative value in the opposite direction. For example, $F S_{i j}^{\max }$ time lag can be interpreted as $S F_{i j}^{\text {min }}$. The objective function (2) minimizes the total graph duration if each vertex will be visited only one time according to precedence relations and topological ordering; constraints (3) assign exactly one execution mode (edge) to each activity performance; constraints (4)-(7) denote precedence relations between vertices as well as time-lags between start-finish times of vertices which can happen due to fuzzy duration estimation of project activities; and finally constraint (8) shows resource availability at each time period $t$. It should be noted that the binary variable $x_{i m t}$ is defined only over the interval between the earliest and latest starting time of each vertex. A time-feasible schedule ST, for generalized precedence relations exists iff generalized precedence relation graph has no cycle of positive length. A schedule which satisfies the resource constraints is called resource-feasible and denoted by SR. A schedule which is both time-feasible and resource feasible is called feasible, and statement $S T \cap S R$ shows the set of feasible schedules. The problem of finding a feasible schedule for the RCPSP and MRCPSP with generalized precedence temporal relations is NP-complete.

\section{$3 \quad$ Fuzzy preferences, decision making under Z-numbers estimations}

Decision making under uncertain environment is still a challenging problem due to the necessity to formalize expert estimations, sometimes even in incomplete probability conditions. Let's consider an ordered pair $(A, B)$, where $A$ plays the role of a fuzzy restriction and component $B$ is the reliability of the first component. Then according to L. Zadeh definition [21] Z-number can be used to represent the information about an uncertain variable of the type, where A represents a value of the variable and $B$ represents an idea of certainty or probability. It is especially important when we need to provide some information about the value of the associated variable as a part of expert estimation and the nature of underlying uncertainty. A simple example of Z-number is given in Figure 3. 


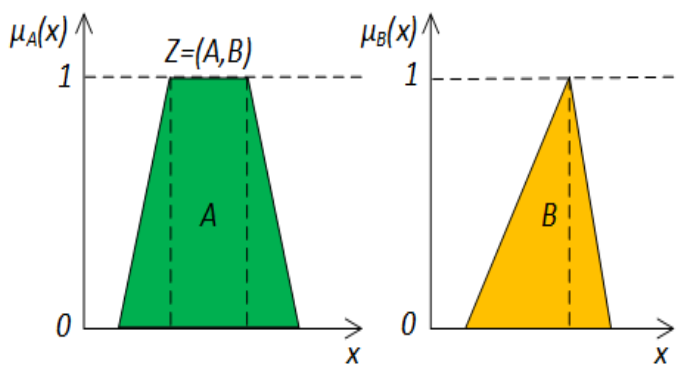

Figure 3: A Z-number example

Definition 5. Let a fuzzy set $A$ be defined on a universe $X$ and be given $A=\left\{\left\langle x, \mu_{A}(x)\right\rangle, x \in X\right\}$, where $\mu_{A}: X \rightarrow[0,1]$ is the membership function $\mathrm{A}$. The fuzzy decision-maker expectation of a fuzzy set $A$ is denoted as:

$$
E_{A}(x)=\int x \mu_{A}(x) d x
$$

The membership function $\mu_{a}(x)$ here provides the degree according to which variable $x \in X$ belongs to set A. The fuzzy expectation of a fuzzy set can be interpreted as the Information Strength supporting the fuzzy set A. In real multi-criteria decision-making problems (MCDM) the domain expert may give fuzzy estimations, so Z-numbers can describe both a restraint and reliability.

In this paper we apply Z-numbers to multi-criteria optimization decision-making problem as a frame to make rational decisions based on uncertain, imprecise and incomplete input information. The concept of Znumbers is used to present decision makers reliability about an uncertain variable of the type where A represents a value of the variable $\mathrm{X}$, and the second component, B represent an idea of certainty or probability such as the concept of sureness, confident, reliability, strength of belief and possibilities.

\subsection{Mode assignment problem}

The main idea of assigning execution modes for activities on graph structure for MRCPSP is based on construction of fuzzy decision-making matrix or socalled judgment matrix. In literature there are several approaches to handle fuzzy MCDM problems. Several are based on fuzzy TOPSIS method (Technique for Ordering of Preference by Similarity to Ideal Solution) and its modifications [17, 4]. TOPSIS method is used to represent the importance of criteria, rating of the alternative and is based on comparing the expected cost criteria of each alternative against the expected benefit criteria to see whether the benefits outweigh the costs and for how much. Recent researches on TOPSIS with multiple fuzzy rule bases (MFS-TOPSIS) [19] and Znumbers fuzzy rule based approach (Z-FRBS TOPSIS)
[18] introduced the influence degree of each decisionmaker in case one decision-maker has more knowledge of the domain and can obtain greater degree of importance comparing with others.

In this paper we consider some ideas of TOPSISs first steps in order to construct a decision matrix and to show the importance of criteria time-resource trade-off and alternative mode preference, as well as Z-number reliability of decision-maker.

In the first step we evaluate the opinion of decisionmaker and assume that there are m-alternative modes for each activity execution in Multi-mode Resourceconstrained Project Scheduling Problem (MRPSP). Lets denote a set of alternative modes as $M_{1}, M_{2}, \ldots M_{n}$ and a number of n-criteria $C_{1}, C_{2}, \ldots, C_{n}$ (here $\mathrm{n}=2$, and they present time vs renewable resource trade-off for each alternative mode). Modified algorithm for activity mode evaluation and initial mode assignment can be presented as several steps of TOPSIS technique as follows:

Step 1: Construct Z-number estimation using membership functions $\mathrm{A}$ and $\mathrm{B}$, converting reliability to type-1 fuzzy number using expectation and algebraic integral $[8]$ as follows:

$$
\alpha=\frac{\int x \mu_{B} d x}{\int \mu_{B} d x} .
$$

Then add the weight of the second part (reliability) to the first Z-number part of restriction. The weighted Z-number can be denoted as:

$$
\tilde{Z}^{\alpha}=\left\{\left(x, \mu_{\tilde{A}^{\alpha}}\right) \mid \mu_{\tilde{A}^{\alpha}}(x)=\alpha \mu_{\tilde{A}}(x), x \in[0,1]\right\}
$$

and thus can be interpreted as type-1 fuzzy number. It was proven that $\tilde{Z}^{\prime}$ has the same fuzzy expectation with $\tilde{Z}^{\alpha}$ :

$$
\begin{array}{r}
\tilde{Z}^{\prime}=\left\{<x, \mu_{\tilde{Z}^{\alpha}}(x)>\mid \mu_{\tilde{Z}^{\alpha}}(x)=\mu_{\tilde{A}}\left(\frac{x}{\sqrt{a}}\right),\right. \\
x \in[0,1]\}
\end{array}
$$

Step 2: Construct decision matrix $\tilde{M}$ and fuzzy preference matrix $\tilde{P}$. We assume that a decision group may have several experts for each activity mode estimation and taking into consideration the objective function (8) from zero-one mathematical formulation we can we consider the importance of the activity execution time criteria and rate alternatives with respect to each criterion.

Mode assignment problem for each edge can be expressed in matrix form as follows:

$$
\tilde{M}=\left[\begin{array}{cccc}
\tilde{m}_{11} & \tilde{m}_{12} & \ldots & \tilde{m}_{1 l} \\
\tilde{m}_{21} & \tilde{m}_{22} & \ldots & \tilde{m}_{2 l} \\
\ldots & \ldots & \ldots & \ldots \\
\tilde{m}_{n 1} & \tilde{m}_{n 2} & \ldots & \tilde{m}_{n l}
\end{array}\right],
$$




$$
\tilde{P}=\left[\begin{array}{llll}
\tilde{p}_{1} & \tilde{p}_{2} & \ldots & \tilde{p}_{l}
\end{array}\right],
$$

where $\tilde{m}_{n l}$ denotes a certain mode assigned for $n$ activities of the project, $\tilde{m}_{n l}=\left(\tilde{d}_{i}, r_{i}\right), l$ is an index number of mode and $\tilde{p}_{l}$ denotes a mode preference for $n$-th activity, that can be described by fuzzy number. Classical TOPSIS approach usually apply normalization procedure for the fuzzy decision matrix $\tilde{M}$ in order to make various scales comparable and apply linear scale transformation as benefit/cost trade-off to obtain. Resource-constraint scheduling problem by contrast assumes optimization between time and resources or so-called costs of the project, so here we will consider priority for each alternative execution mode, that is based on the preference model.

A crisp preference $\mathrm{P}$ is a binary relation that reflects the certainty of the preference between two alternative modes. For example, having two modes $m_{1}$ and $m_{2}$ for activity 1 the statement $m_{11} \succ m_{12}$ means that $m_{11}$ is strictly more preferable for decision maker than $m_{12}$, while $m_{11} \sim m_{12}$ means indifference.

Definition 5. Let $\tilde{M}_{i}=\left\{\tilde{m}_{1}, \tilde{m}_{2}, \ldots, \tilde{m}_{l}\right\}$ be the set of alternative modes for $i$-th project activity. A fuzzy preference over $\tilde{M}_{i}$ is a fuzzy binary relation on $\tilde{M}_{i}$, represented by preference matrix $\tilde{P}$ with membership function $\mu_{p}: M \times M \rightarrow[0,1]$, where $\mu_{p}\left(m_{1}, m_{2}\right)=p_{12}$ is treated as the degree of preference for mode $m_{1}$ over mode $m_{2}$.

Further fuzzy interpretation allows to conclude the following states:

$p\left(m_{1}, m_{2}\right)=1$ indicates that mode $m_{1}$ is definitely preferred to $m_{2}$;

$p\left(m_{1}, m_{2}\right)>0.5$ indicates that mode $m_{1}$ is likely to be preferred to $m_{2}$;

$p\left(m_{1}, m_{2}\right)=0.5$ indicates that mode $m_{1}$ is likely to be indifferent to $m_{2}$;

$p\left(m_{1}, m_{2}\right)<0.5$ indicates that mode $m_{2}$ is likely to be preferred to $m_{1}$;

$p\left(m_{1}, m_{2}\right)=0$ indicates that mode $m_{2}$ is definitely to preferred to $m_{1}$;

Step 3: For each directed edge $e \in E$ in graph, where $E=\left\{e_{1 m}, e_{2 m}, \ldots, e_{n m}\right\}$ sort modes according to fuzzy degree of preference. Make priority list.

\subsection{Geometrical interpretation of rectangle packing for F-MRCPSP with mode-dependent time intervals}

Some of operations research problems, especially network flow programming models, transportation and assignment problems, packing and scheduling problems can be geometrically interpreted and some similarities can be found out. Visualizing fuzzy graph scheduling problem as the packing one, we need to use three dimensions: membership degree, time intervals and resources correspondingly. A simple example of rectangle packing illustration of F-MRCPSP is given on Figure 4. An empty rectangle box should

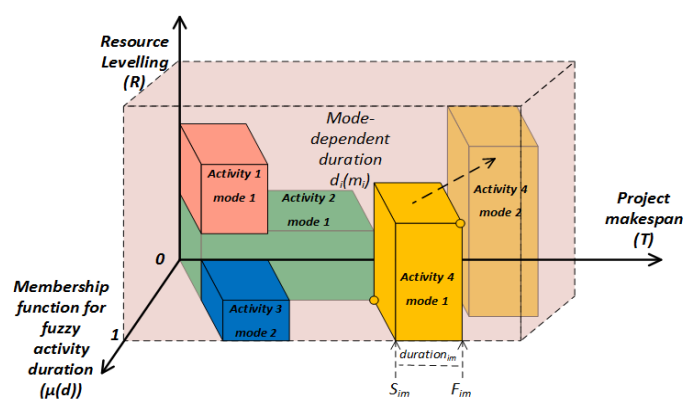

Figure 4: Rectangle packing illustration

be packed with a number of activities (boxes) to be scheduled. Thus we have a triple $(T, R, \mu(d))$, where for each $\left(x_{i}, y_{i}, z_{i}\right)$ the coordinates of each n-box can be determined as follows: $\left(x_{i}+d_{i}, y_{i}+r_{i}, \mu d_{i}\right)$, where $i=1, . ., n$, and the finishing time of final box (activity) $x_{i}+d_{i}$ should not exceed $T$. The only extra constraint is a precedence requirements for the activity execution. The objective function of such interpretation of F-MRCPSP is to minimize the total makespan $T$, while the objective function of classical rectangle packing problem is to maximize the total area usage.

\section{Decision methodology for F-MRCPSP}

The objective of the basic MRCPSP is to construct a feasible/optimal schedule of activity performance, i.e. start and finish times within execution mode selection, so that precedence as well as resource constraints are satisfied while minimizing the total project makespan T. The multi-mode resource-constrained project scheduling problem (MRCPSP) can be formally stated as a number of single-mode instances. The efficient way to solve this type of a problem stated in (1)-(9) is to split the problem into two sub-problems: mode-assignment problem and single mode project scheduling step. Usually mode assignment step is solved by Satisfiability problem solver or Boolean satisfiability problem (SAT) [6] and it returns a feasible mode selection, while single-mode project scheduling problem can be solved using different meta-heuristic procedures. The Boolean satisfiability problem (SAT) is, given a formula, to check whether it is satisfiable or not. A formula is said to be satisfiable if it can be made TRUE by assigning appropriate logical values (i.e. TRUE if the mode was assigned to activity, FALSE-otherwise) to its variables, but it does not consider fuzzy nature of decision process, as well as preferences of decision-makers toward some execution modes.

In our approach we use mode assignment procedure 
from subsection 3.1 of the paper to construct priority list according to three decision-making steps. Scheduling step is based on precedence tree-based approach and assumes an enumeration scheme that evaluates all possible partial feasible schedules [5] as well as bounding rules and the possibility to dynamically switch modes from priority list.

Let's consider the following notations for precedence tree-based algorithm:

$l, i_{l}$ - level $l$ of enumeration tree, activity $i$ at $l$-level; $m_{i l}$ - mode selected from priority list $\tilde{P}$ for activity $i$ at $l$ level;

$\tilde{S}_{i} ; \tilde{F}_{i}$ - fuzzy start and finish times of activity $i$

$\tilde{d}_{i m}$ - fuzzy duration of activity $i$ performed in mode $m ; t$ - discrete time unit;

$S c h V_{l}$ - set of already scheduled activities at $l$-level of enumeration tree;

Suit $V_{l}$ - set of suitable (active) to scheduling activities at $l$-level, i.e. vertices whose immediate predecessors were completely finished;

$e s_{i}$ - earliest feasible start time of activity $i$

Pred $_{i}$ - set of predecessor activities of $i$-activity;

$S u c c_{i}$ - set of successor activities of $i$-activity;

Then precedence tree-based branch-and-bound algorithm based on enumeration scheme based on partial schedules can be represented as follows:

\section{Step 1: Initialization.}

Set $\mathrm{l}=1$; starting activity $\mathrm{i}=1 ; \mathrm{t}=0 ; m_{i 1}=1 ; S \operatorname{ch} V_{l}=\emptyset$;

Step 2: Updating sets of activities.

Increase tree level: $l=l+1$.

Update set of scheduled activities:

$S \operatorname{ch} V_{l}=S \operatorname{ch} V_{l-1} \cup\left\{i_{l-1}\right\}$;

Compose the set of suitable activities:

SuitV $_{l}=\left\{i \in V \backslash\right.$ SchV $_{l} \mid$ Pred $\left._{i} \subset \operatorname{Sch}_{l}\right\}$

If the last dummy activity is active: $n+1 \in$ Suit $_{l}$, then store the current solution and go to Step 5. Else go to step 3.

Step 4: Selecting a mode for selected activity and fuzzy scheduling.

If there is no untested mode left in priority list for $\left\{1, \ldots, \tilde{M}_{i l}\right\}$ for activity $i$, then go to step 3 ,

Else select next untested mode from priority list:

$m_{i l} \in\left\{1, \ldots, \tilde{M}_{i l}\right\}$;

Compute the earliest precedence feasible start time:

$e s_{i l}=\max \left\{\tilde{F}_{i l} \mid i \in\right.$ Pred $\left._{i l}\right\}+1$;

Compute start time $\tilde{S}_{i l}$ and finish time $\tilde{F}_{i l}$ for each $(i, j) \in V$;

\section{Step 5: Backtracking.}

Decrease the precedence tree level by $1: l=l-1$.

If the precedence tree level $l=1$, then STOP. Else go to Step 4.

The algorithm allows to evaluate all possible partial schedules during the operational stage of planning and choose feasible final schedule for activity execution.

\section{Conclusion and discussion}

In this paper the approach to formalize temporal uncertainty in graph structure and fuzzy multi-mode resource-constrained project scheduling problem was considered. This approach splits the problem into mode assignment problem and scheduling step. It also considers mode-dependent fuzzy time intervals between activities and fuzzy preference relations for mode selection step which increase the complexity of approach. It also allows some flexibility in scheduling towards temporal precedence relations in graph for decision-making process. Geometrical interpretation of F-MRCPSP as rectangle packing problem is presented, and an idea of reducing some problems of operational research to discrete structures and graph models is given. The first formal steps of TOPSIS method for decision-making process is adopted to solve mode-assignment problem and algorithm for finding feasible schedule is suggested.

\section{Acknowledgement}

This work has been supported by the Ministry of Education and Science of the Russian Federation under Project "Methods and means of decision making on basis of dynamic geographic information models" (Project part, State task 2.918.2017).

\section{References}

[1] J. Allen, Maintaining knowledge about temporal intervals, Communications of the ACM 26(11) (1983) 832-843.

[2] A. Bozhenyuk, S. Belyakov, J. Kacprzyk, Optimization of jobs in GIS by coloring of fuzzy temporal graph, in: Advances in Intelligent Systems and Computing (ICAFS'2018), Vol. 896, Springer-Verlag Berlin Heidelberg, 2019, pp. 2532.

[3] A. Bozhenyuk, S. Belyakov, M. Knyazeva, I. Rozenberg, Searching method of fuzzy internal stable set as fuzzy temporal graph invariant, in: Communications in Computer and Information Science (IPMU'2018), Vol. 853, Springer-Verlag Berlin Heidelberg, 2018, pp. 501-510.

[4] C.-T. Chen, Extensions of the topsis for group decision making under fuzzy environment, Fuzzy Sets and Systems 114(1) (2000) 1-9.

[5] J. Cheng, J. Fowler, K. Kempf, S. Mason, Multi-mode resource-constrained project scheduling problems with non-preemptive activity splitting, Computer and Operations Research 53 (2015) 275-287. 
[6] J. Coelho, M. Vanhoucke, Multi-mode resourceconstrained project scheduling using rcpsp and sat solvers, European Journal of Operational Research 213(1) (2011) 73-82.

[7] C. Dutta, G. Pandurangan, R. Rajaraman, Z. Sun, E. Viola, On the complexity of information spreading in dynamic networks, in: Proceedings of the 24th Annual ACM-SIAM Symposium on Discrete Algorithms (SODA'2013), 2013, pp. 717-736.

[8] B. Kang, D. Wei, Y. Li, Y. Deng, A method of converting z-number to classical fuzzy number, Journal of Information and Computational Science 3 (2012) 703-709.

[9] A. Kaufmann, Introduction a la theorie des sonsensembles flous, Masson Paris 1 (1973) 41-189.

[10] F. Kuhn, N. Lynch, R. Oshman, Distributed computation in dynamic networks, in: In Proceedings of the 42nd ACM symposium on Theory of computing (STOC'10), New York, NY, USA, 2010, pp. 513-522.

[11] J. P. M. Blue, B. Bush, Unified approach to fuzzy graph problems, Fuzzy Sets and Systems 125(3) (2002) 355-668.

[12] G. Mertzios, O. Michail, I. Chatzigiannakis, P. G. Spirakis, Temporal network optimization subject to connectivity constraint, in: 40th International Colloquium on Automata, Languages and Programming (ICALP'2013), Vol. 7966 of Lecture Notes in Computer Science, Springer-Verlag Berlin Heidelberg, 2013, pp. 657-668.

[13] O. Michail, P. G. Spirakis, Traveling salesman problems in temporal graphs, in: E. Csuhaj-Varj, M. Dietzfelbinger, Z. sik (Eds.), In 39th International Symposium on Mathematical Foundations of Computer Science (MFCS'14), Mathematical Foundations of Computer Science 2014, SpringerVerlag Berlin Heidelberg, 2014, pp. 553-564.

[14] J. N. Mordeson, S. Mathew, D. S. Malik, Fuzzy Graph Theory with Applications to Human Trafficking, Vol. 365 of Studies in Fuzziness and Soft Computing, Springer, Switzerland, 2018.

[15] B. D. Reyck, W. Herroelen, The multi-mode resource-constrained project scheduling problem with generalized precedence relations, European Journal of Operational Research 119 (1999) 538556.

[16] A. Rosenfeld, Fuzzy graphs, in: L. A. Zadeh, K. S. Fu, M. Shimura (Eds.), Fuzzy Sets and Their Applications, Academic Press, 1975, pp. 77-95.
[17] F. J. J. Santos, H. Carmago, Decision support systems in multicriteria groups: An approach based on fuzzy rules, in: Proceedings IEEE International Conference on Fuzzy Systems (FUZZ-IEEE 2010), Barcelona, Spain, 2010, pp. 1-8.

[18] A. M. Yaakob, A. Gegov, Fuzzy rule based approach with z-numbers for selection of alternatives using topsis, in: Proceedings IEEE International Conference on Fuzzy Systems (FUZZ-IEEE 2015), Istanbul, Turkey, 2015, pp. 1-8.

[19] A. M. Yaakob, A. Gegov, M. Bader-El-Den, S. A. Rahman, Fuzzy systems with multiple rule bases for selection of alternatives using topsis, in: Proceedings IEEE International Conference on Fuzzy Systems (FUZZ-IEEE 2016), Vancouver, BC, Canada, 2016, pp. 1-8.

[20] R. T. Yeh, S. Bang, Fuzzy relations, fuzzy graphs, and their applications to clustering analysis, in: L. A. Zadeh, K. S. Fu, M. Shimura (Eds.), Fuzzy Sets and Their Applications, Academic Press, 1975, pp. 125-149.

[21] L. A. Zadeh, A note on z-numbers, Information Sciences 181 (2011) 2923-2932. 